\title{
Seminare / Séminaires / Seminari 2008
}

\section{Praxiseröffnung/-übernahme}

Themen (Details $\rightarrow$ www.fmhservices.ch)

Juristische Aspekte - Ehe- und Erbrecht - Praxiseinrichtung - Praxisadministration - Unternehmensbewertung einer Arztpraxis - Finanzierung einer Arztpraxis - Versicherungen.

\section{Sponsoren}

Versicherung der Schweizer Ärzte, Schweizerische Ärzte-Krankenkasse, Druckerei Schmid-Mogelsberg, Labornetz daCapo, Unilabs SA, Labor medica, Labor team w ag, Kern Concept AG und EMH Schweizerischer Ärzteverlag AG.

\section{Daten}

$\begin{array}{llll}\text { K04 } & \begin{array}{l}\text { Donnerstag, 4. September 2008 } \\ \text { Zürich }\end{array} & \text { FMT } \\ \text { K05 } & \begin{array}{l}\text { Donnerstag, 6. November 2008 } \\ \text { Basel }\end{array} & \begin{array}{l}\text { Hotel } \\ \text { 9.00-16.30 Uhr }\end{array} & \begin{array}{l}\text { Victoria } \\ \text { Kanr }\end{array}\end{array}$

\section{Praxisübergabe}

\section{Hinweis}

Vor allem aus steuerplanerischer Sicht lohnt es sich, sich bereits frühzeitig (5-10 Jahre) mit diesem Thema auseinanderzusetzen.

\section{Themen (Details $\rightarrow$ www.fmhservices.ch)}

Juristische Aspekte - Unternehmensbewertung einer Arztpraxis - Versicherungen - Steuern.

\section{Sponsoren}

Versicherung der Schweizer Ärzte, Schweizerische Ärzte-Krankenkasse, Labor medica, Labor team w ag, Kern Concept AG und EMH Schweizerischer Ärzteverlag AG.

\section{Daten}

$\begin{array}{llll}\text { K09 } & \begin{array}{l}\text { Donnerstag, 11. September 2008 } \\ \text { Zürich }\end{array} & \text { FMT } \\ \text { 13.30-18.00 Uhr } & \\ \text { K10 } & \begin{array}{l}\text { Donnerstag, 13. November 2008 } \\ \text { Basel }\end{array} & \begin{array}{l}\text { Hotel } \\ \text { 13.30-18.00 Uhr }\end{array} & \text { Victoria }\end{array}$

\section{Finanz- und Steuerplanung}

\section{Themen (Details $\rightarrow$ www.fmhservices.ch)}

Finanzplanung (Businessplan, buchhalterische Massnahmen vor Praxiseröffnung/-übernahme, Standardkontenplan für Ärzte, System der doppelten Buchhaltung, EDV-unterstützte Buchfüh- rungslösung), Steuerplanung (Steueraspekte bei Eintritt in die Selbständigkeit, Steuerfallen und Steuerrisiken, optimierte Steuerplanung).

\section{Sponsoren}

Kern Concept AG und EMH Schweizerischer Ärzteverlag AG.

\section{Daten}

\begin{tabular}{lll} 
K13 & $\begin{array}{l}\text { Donnerstag, 18. September 2008 } \\
\text { Zürich }\end{array}$ & FMT \\
13.30-18.00 Uhr & \\
K14 & $\begin{array}{l}\text { Donnerstag, 20. November 2008 } \\
\text { Basel }\end{array}$ & $\begin{array}{l}\text { Hotel } \\
\text { 13.30-18.00 Uhr }\end{array}$ \\
\hline
\end{tabular}

\section{Praxiscomputerworkshop}

\section{Inhalt}

Die Workshopteilnehmer/-innen erhalten im 1. Teil eine Einführung in die Anforderungen an ein Praxisinformationssystem. Anhand einer modernen vernetzten Praxisinfrastruktur werden die Beurteilungskriterien für eine praxisund zukunftstaugliche Softwarelösung dargestellt. Checklisten sollen die schnelle Orientierung unterstützen und bei der Beurteilung und Wahl des Produkts konkrete Hilfe bieten. In Zusammenarbeit mit SGAM.Informatics werden die zentralen Elemente der elektronischen Krankengeschichte aufgezeigt. Ein Erfahrungsbericht eines EDV-Anwenders (Arzt) rundet den 1. Teil ab. Der 2. Teil umfasst die Präsentation von sechs Praxisadministrationssoftwarelösungen (Leistungserfassung, elektronisches Abrechnen unter Einbezug der TrustCenter, Agendaführung, Statistiken, Laborgeräteeinbindung, elektronische Krankengeschichte, Finanzbuchhaltungslösungen usw.).

\section{Ziel}

Die Workshopteilnehmer/innen erhalten einen Anforderungskatalog, der ihnen erlaubt, ihre Vorstellungen von einem modernen Praxisinformationssystem besser $\mathrm{zu}$ formulieren und diese dem Softwarehersteller zu dessen Vorbereitung zu kommunizieren. Zudem erhalten sie einen ersten Überblick über führende Softwarelösungen.

\section{Daten}

K17 $\begin{array}{ll}\text { Donnerstag, } 27 . \text { November } 2008 & \text { Stadt- } \\ \text { theater }\end{array}$ 


\section{Ouverture et reprise d'un cabinet médical}

Contenu (Détails $\rightarrow$ www.fmhservices.ch)

Business plan - Aménagement - Estimation d'inventaire et goodwill d'un cabinet - Administration d'un cabinet médical - Assurances Passage du statut de salarié à celui d'indépendant et fiscalité.

\section{Sponsors}

Assurance des Médecins Suisses, Caisse-Maladie des Médecins Suisses, Unilabs SA, Viollier SA, Labor team w sa, MCL Medizinische Laboratorien SA et EMH Editions médicales suisses SA.

\section{Dates}

$\begin{array}{lll}\text { K22 Jeudi } 4 \text { septembre } 2008 & \text { Ramada } \\ \text { Genève } \quad 17.00-21.00 ~ h & \text { Park Hôtel }\end{array}$

\section{Remise d'un cabinet médical}

\section{Contenu (Détails $\rightarrow$ www.fmhservices.ch)}

Aspects juridiques - Estimation d'inventaire et goodwill d'un cabinet - Assurances - Conséquences fiscales d'une remise.

\section{Sponsors}

Assurance des Médecins Suisses, Caisse-Maladie des Médecins Suisses, Labor team w sa et EMH Editions médicales suisses SA.

\section{Dates}

$\begin{array}{lll}\text { K25 Jeudi } 13 \text { novembre } 2008 & \text { Hôtel } \\ \text { Genève } \quad 17.00-20.45 \text { h } & \text { Royal }\end{array}$

\section{Contabilità e fiscalità (Ticino)}

\section{Contenuto}

L'obiettivo di questo seminario è di dare al medico le conoscenze necessarie per utilizzare autonomamente lo strumento contabile e per operare con più efficienza ed economicità, nonché far comprendere al medico il significato della pianificazione fiscale.

Mediante esempi pratici elaborati in gruppo, sarà illustrato in maniera semplice, ma completa il sistema di gestione della contabilità di uno studio medico con effetto anche sulla pianificazione fiscale.

\section{Dates}

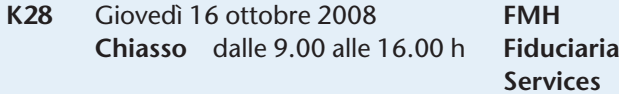

\section{Anmeldung und Auskunft / Inscription et information}

Internet unter www.fmhservices.ch oder FMH Consulting Services, Sandra Stadelmann, Burghöhe 1, 6208 Oberkirch, Tel. 04192500 77, Fax 0419210586

\section{Hinweis/Remarque}

Bei sämtlichen Seminaren, bei denen die Kosten teilweise oder gänzlich von Seminarsponsoren gedeckt werden, werden die Teilnehmeradressen den jeweiligen Sponsoren zur Verfügung gestellt. Les adresses des participants aux séminaires dont les coûts sont couverts en partie ou totalement par des sponsors sont communiquées aux sponsors concernés.

\section{Annullierungsbedingungen / Conditions d'annulation}

Bei Abmeldungen oder Fernbleiben werden folgende Unkostenbeiträge erhoben:

Un montant est perçu pour une absence ou une annulation. Il est de:

- Fr. 50.- pro Person ab 14 Tage vor Seminarbeginn / par personne dans les 15 jours avant le début du séminaire;

- Fr. 80.- pro Person ab 7 Tage vor Seminarbeginn oder Fernbleiben / par personne dans les 7 jours avant le début du séminaire. 


\section{Seminarsponsoren 2008}

Die Unterstützung durch verschiedene Sponsoren ermöglicht es der FMH Consulting Services AG, ihre Seminarreihen für FMH-Mitglieder teils kostenlos, teils kostengünstig anzubieten. Gerne stellen wir Ihnen diese Firmen in einem Kurzporträt vor.

\section{daCapo}

info@dacapo.ch

www.dacapo.ch

daCapo ist eine lose Interessengemeinschaft von vier eigenständigen medizinischen Labors, die mit Arztpraxen eine sehr persönliche und unkomplizierte, von Sicherheit, Qualität und Effizienz geprägte Zusammenarbeit pflegen. Bei uns stehen Sie immer in direktem, kollegialem Kontakt mit den Laborinhabern und den leitenden Mitarbeiterinnen und Mitarbeitern, die Ihre Analyseaufträge von A bis Z persönlich betreuen. MLO Olten (www.mlo.ch) • Rothen Medizinische Laboratorien, Basel (www.laborrothen.ch) - Medics Labor AG, Bern (www. medics-labor.ch) • Laborgemeinschaft 1 (LG1), Zürich (www.lg1.ch).

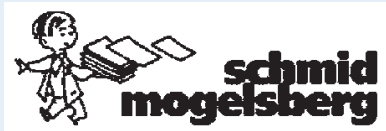

P. Schmid + Co. AG, Ärztedrucksachen Sonnmattstrasse 1, 9122 Mogelsberg

Tel. 07137560 80, Fax 0713756081

info@schmid-mogelsberg.ch

www.schmid-mogelsberg.ch

\section{Seit 70 Jahren auf Ärztedrucksachen spezialisiert!}

Bei der Gestaltung von zweckmässigen Arztformularen (Patientenkarten, Labor- und Statusformulare usw.) profitieren Sie von der langjährigen Erfahrung. Die Vergangenheit hat gezeigt, dass sich kaum zwei Ärzte für den gleichen Druck entscheiden. Zweckmässige Materialauswahl, einwandfreie Verarbeitung, freundliche und kompetente Beratung, schnelle Lieferung und die Ausführung von Spezialwünschen diese Dienstleistungen schaffen die Grundlage für ein langjähriges Vertrauensverhältnis. Auf Wunsch versenden wir eine individuell auf die Fachrichtung zusammengestellte Druckmusterkollektion.
An über 9000 Ärzte liefern wir Drucksachen, Papiere und Büroartikel für den Praxisalltag.

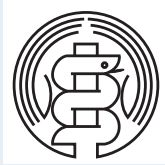

Schweizerische Ärzte-Krankenkasse Oberer Graben 37, 9001 St. Gallen Tel. 07122718 18, Fax 0712271828 info@saekk.ch www.saekk.ch

Die richtige Adresse für Erwerbsausfalldeckungen, Kollektivkrankenkasse und Versicherungsplanung

Mit mehr als 100 Jahren Erfahrung kennt unsere Organisation auch heute die Bedürfnisse der Ärztinnen und Ärzte. Sie bietet entsprechend durchdachte und kostengünstige Lösungen an, sowohl für Praxiseröffner/-innen wie auch für selbständige und angestellte Ärztinnen und Ärzte.

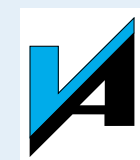

Versicherung der Schweizer Ärzte

Genossenschaft

Länggassstrasse 8, 3000 Bern 9

Tel. 03130125 55, Fax 0313025156

versa@versa.ch

www.versa.ch

Spezialisiert auf die Bedürfnisse von Ärztinnen und Ärzten und deren Ehegatten, bietet die Versicherung der Schweizer Ärzte Genossenschaft individuelle, den jeweiligen Bedürfnissen angepasste Versicherungslösungen im Bereich der privaten Vorsorge an.

\section{(I) Unilabs}

www.unilabs.ch

\section{UNILABS - Ihr Schweizer Qualitätslabor} schnell, zuverlässig und nah

Unilabs ist im Bereich der medizinischen Analysen ein kompetenter, transparenter und zuverlässiger Partner. Wir bieten Ihnen überall in der Schweiz ein komplettes Analysenspektrum, umfassende Dienstleistungen und kompetente 
Fachberatung an. In der Deutschschweiz sind dies Unilabs Mittelland mit den Standorten Bern, Burgdorf, Langnau, Solothurn; Unilabs Zürich und Unilabs Dr. Weber.

Unilabs bietet nicht nur medizinische Laboranalysen, sondern auch wertvolle individuelle Zusatzdienstleistungen wie Beratung und Weiterbildung für alle Mitglieder des Praxisteams, Unterstützung bei der nachhaltigen Reduktion des Praxisaufwandes und Beratung bei Praxisund Laborbedarf.

Die Unilabs-Strategie zielt darauf ab, ihre vielfältigen und regionalen Dienstleistungen über eine fundierte wissenschaftliche Struktur sicherzustellen. Zur Gewährleistung einer hochstehenden Qualität werden stets die notwendigen Investitionen für Ausrüstung und Ausbildung getätigt.

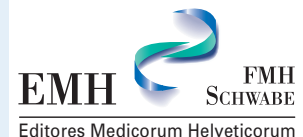

EMH Schweizerischer Ärzteverlag AG Farnsburgerstrasse 8, 4132 Muttenz Tel. 06146785 55, Fax 0614678556 verlag@emh.ch www.emh.ch

EMH, der Verlag der Ärztinnen und Ärzte Die EMH Schweizerischer Ärzteverlag AG wurde 1997 gegründet. EMH ist ein Gemeinschaftsunternehmen der Verbindung der Schweizer Ärztinnen und Ärzte FMH und der Schwabe AG, Basel, des mit Gründung 1488 ältesten Druckund Verlagshauses der Welt.

Hauptpublikationen von EMH sind die Zeitschriften «Schweizerische Ärztezeitung», das offizielle Publikationsorgan der FMH, «Swiss Medical Forum» mit praxisorientierten Fortbildungsbeiträgen sowie "Swiss Medical Weekly», die Plattform für klinisch orientierte Wissenschaftler. Ebenfalls zu den Hauptpublikationen zählt «PrimaryCare», das ab 2008 unter neuer Herausgeberschaft als offizielle «Schweizerische Zeitschrift für Hausarztmedizin» weiterhin bei EMH erscheint.

Als erfolgreiches Onlineangebot ist unter anderem die Fortbildung des «Swiss Medical Forum» unter www.smf-cme.ch zu nennen. Steigende Zugriffszahlen und die Akkreditierung durch die Fachgesellschaften SGAM und SGIM als strukturierte und nachweisbare Fortbildung belegen diesen Erfolg.

Weitere medizinische Fachzeitschriften, ein ständig wachsendes Buchprogramm sowie viele
Kooperationen und Dienstleistungen runden das umfangreiche Verlagsangebot ab.

\section{medica \\ MEDIZINISCHE LABORATORIEN Dr. F. KAEPPEUL AG}

\section{MEDIZINISCHE LABORATORIEN}

DR. F. KAEPPELI AG

Eidgenössisch anerkannte Laboratorien

Wolfbachstrasse 17, 8024 Zürich

Tel. 04426999 99, Fax 0442699909

info@medica-labor.ch

www.medica-labor.ch

Der promovierte Mikrobiologe und Biochemiker Dr. F. Käppeli, Laborspezialist FAMH, übernahm 1976 das heute 50jährige Unternehmen und gründete als dessen Leiter und Inhaber die Einzelfirma medica. Der wichtigste unternehmerische Leitgedanke von Dr. F. Käppeli heisst kontinuierliche Innovation und Schaffung wegweisender Standards auf allen Gebieten der Labormedizin: Mikrobiologie inklusive Parasitologie, Serologie, Immunologie, klinische Chemie, Hämatologie, molekulare Diagnostik und Pathologie in Human- und Veterinärmedizin. So entstand ein Kompetenzzentrum für Labordiagnostik von gesamtschweizerisch grosser Bedeutung. Die modernst ausgebauten Laboratorien werden laufend erweitert und befinden sich im Herzen von Zürich. Gegen 200 Angestellte der Partnerlabors, begleitet von Spezialisten aus Medizin, Pharmakologie, Naturwissenschaften und Technik, garantieren für höchste Professionalität.

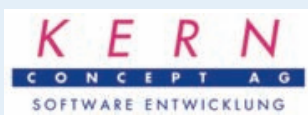

Kern Concept AG

Wiler Strasse 75, 9200 Gossau SG

Tel. 07138880 30, Fax 0713888031

info@kernconcept.ch

www.kernconcept.ch

KERN CONCEPT AG entwickelte und betreut das Administrationsprogramm AESKULAP, das heute in rund 1200 Arztpraxen erfolgreich eingesetzt wird. AESKULAP ist ein extrem schnelles Windows-Programm, das zusammen mit einer SQL-Datenbank höchste Verarbeitungsgeschwindigkeit, Kompaktheit und schnellste Zugriffsmöglichkeiten gewährleistet. AESKULAP bietet für alle Bedürfnisse eine optimale Lösung: vom einfachen Abrechnungssystem mit sämtlichen Leistungserfassungs- 
möglichkeiten bis zur vollstrukturierten elektronischen KG-Führung, die in der Praxis keine Wünsche offenlässt. AESKULAP ist ein modular aufgebautes, anpassbares System mit einer Vielzahl von Modulen. Auch verfügt es über einen integrierten TARMED-Validator sowie einen TARMED-Optimizer. Das gewährleistet vollständige Rechnungen, da technische Grundleistungen, Prozentzuschläge und Alterszuschläge vollautomatisch hinzugefügt werden.

\section{\$ VIOLLIER}

Viollier AG

Spalenring 145/147, 4002 Basel

Tel. 06148611 11, Fax 0614820030

contact@viollier.ch

www.viollier.ch

Unsere Kernkompetenz ist die Labormedizin. Dank optimaler Analysenvielfalt, Qualität und Geschwindigkeit sind wir ein vertrauenswürdiger Partner. Dadurch machen wir Kunden erfolgreicher und sicherer in Prävention, Diagnose und Therapie.

Qualität und Effektivität sind Pfeiler unserer Dienstleistungen. Die durch persönliche Beziehungen geprägte Partnerschaft basiert zum einen auf klinisch fokussierten Darstellungen für die Analysenauswahl, einfachen präanalytischen Hinweisen für den Probentransport und präzisen, rasch übermittelten Resultaten. Zum anderen bieten wir jederzeit kompetente Beratung und permanente Kommunikationsbereitschaft.

Zusätzlich zum Laborcenter sind in Basel Kardiologie und Histopathologie sowie Tochtergesellschaften in Bad Ragaz, Bern, Biel/Bienne, Fribourg, Genève, Lachen, Lausanne, Lugano, Morges, Nyon, Thun, Winterthur, Zug und Zürich angeschlossen.

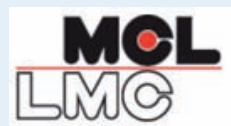

MCL Medizinische Laboratorien AG Freiburgstrasse 634

3172 Niederwangen

Tel. 03132878 78, Fax 0313287880

info@mcl.ch

www.mcl.ch
Die Schweizer Firma MCL Medizinische Laboratorien AG besteht seit 27 Jahren und ist nach wie vor im Besitz der Gründerfamilie Dr. Drescher. Das seit Juni 2000 akkreditierte Unternehmen mit Hauptsitz in Niederwangen bei Bern hat sich als 24-Stunden-Labor mit Routine-, Notfallund Spezialanalytik für zahlreiche Arztpraxen und Spitäler etabliert. Satellitenlabors betreibt die MCL in Bern, Fribourg, Saanen, Winterthur, Lugano und Locarno.

Mit ihren 185 Mitarbeitenden und modernster Laborautomation zeichnet sich die MCL durch Qualität in der Analytik, eine schnelle Verfügbarkeit der Resultate und ausgezeichnete Dienstleistungen aus. Die Wissenschaftler von MCL stehen unseren Auftraggebern als Berater für fachspezifische Fragen der Labormedizin zur Verfügung, denn der Dialog mit jedem einzelnen Kunden und dessen Zufriedenheit mit unseren Leistungen sind für uns von grösster Wichtigkeit.

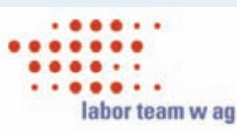

labor team w ag

Blumeneggstrasse 55

9403 Goldach

Tel. 07184445 45, Fax 0718444546

info@team-w.ch

www.team-w.ch

Spezialleistungsspektrum - Dank Zusammenarbeit mit Spezialisten im In- und Ausland können wir ein vollständiges Spektrum der Laboranalytik anbieten. Wir sind bestrebt, Ihnen stets die aktuellsten Laborparameter $\mathrm{zu}$ offerieren. Individualität - Ihre spezifischen patientenbezogenen Fragen beantworten wir schriftlich oder telefonisch. Die interdisziplinäre Bewertung von Analyseergebnissen ist für uns selbstverständlich. Fortbildung - In Zeiten immer modernerer Labordiagnostik bieten wir Ihnen Unterstützung und Informationen in Veranstaltungen und Seminaren über Themen, die von präanalytischer Hilfestellung bis zur diagnostischen Interpretation reichen. Zukunft - Die labor team w ag hat es sich zum Ziel gesetzt, eine praxisnahe Versorgung in der medizinischen Analytik anzubieten. Die langjährige Erfahrung unserer Mitarbeiter garantiert, dass das innovative, qualitativ hochstehende Laborkonzept umgesetzt wird. 